\title{
Immunophenotype of High-Grade Prostatic Adenocarcinoma and Urothelial Carcinoma
}

\author{
Elizabeth M. Genega, M.D., Brian Hutchinson, B.S., Victor E. Reuter, M.D., Paul B. Gaudin, M.D.* \\ Department of Pathology, Memorial Sloan-Kettering Cancer Center, New York, New York and Hospital of \\ the University of Pennsylvania, Philadelphia, Pennsylvania.
}

Morphologic features alone can usually be used to distinguish prostatic adenocarcinoma and urothelial carcinoma of the urinary bladder. Poorly differentiated tumors, however, can occasionally have features of both neoplasms, making determination of site of origin difficult. No study has provided a panel of antibodies to assist in the distinction of these two tumors. For this study, 73 examples of moderately and poorly differentiated prostatic adenocarcinoma and 46 examples of high-grade urothelial carcinoma were obtained from radical resection specimens. Immunohistochemical studies were performed using the following panel of antibodies: cytokeratin (CK) 7, CK 20, 34 $\beta E 12$, Leu M1, carcinoembryonic antigen (CEA)m, CEAp, p53, Leu 7, prostate-specific acid phosphatase (PSAP), prostate-specific antigen (PSA), and B72.3. Mucicarmine was also performed. Intermediate and high-grade prostatic carcinoma were compared and then high-grade prostatic carcinoma was compared with high-grade urothelial carcinoma. PSA and PSAP each stained $94 \%$ of prostatic adenocarcinomas, but no urothelial carcinomas. Leu 7 stained $94 \%$ of prostate and $17 \%$ of urothelial carcinomas. Over half of the urothelial carcinomas showed positivity for $34 \beta \mathrm{E} 12$ (65\%), as did two cases of prostatic carcinoma (6\%). Eighty-three percent of urothelial carcinomas and $12 \%$ of prostatic adenocarcinomas stained with CK 7. Forty-one percent of urothelial carcinomas and $12 \%$ of prostatic carcinomas were reactive for CEAm, and p53 stained $33 \%$ and $3 \%$ of urothelial and prostatic adenocarcinomas, respectively. No significant difference was seen in the expression of CEAp, CK 20, B72.3, Leu M1, or mucicarmine between prostate and

\footnotetext{
Copyright @ 2000 by The United States and Canadian Academy of Pathology, Inc.

VOL. 13, NO. 11, P. 1186, 2000 Printed in the U.S.A.

Date of acceptance: June 2, 2000.

*Current address: Clinical Pathology Associates, 8613 Cross Park Drive Austin, TX 78754-4554.

Presented in part at the annual meeting of the United States and Canadian Academy of Pathology in San Francisco, California, March, 1999.

Address reprint requests to: Elizabeth M. Genega, M.D., Hospital of the University of Pennsylvania, Department of Pathology, 6 Founders, 3400 Spruce Street, Philadelphia, PA 19104; e-mail: genega@mail. med.upenn.edu; fax: 215-349-5910.

urothelial carcinoma. We propose a panel of six antibodies to assist in the distinction of high-grade prostatic adenocarcinoma from high grade urothelial carcinoma: PSA, PSAP, 34 $\beta E 12$, Leu 7, CK 7, and p53. The first three antibodies should be used initially; if results are negative, the remaining antibodies may be employed.

KEY WORDS: 34ßE12; CK 7; immunohistochemistry; Leu 7; prostatic adenocarcinoma; PSA; PSAP; urothelial carcinoma.

Mod Pathol 2000;13(11):1186-1191

Prostatic adenocarcinoma and urothelial carcinoma of the urinary bladder are the most common and fifth most common cancers in men, respectively (1). Both neoplasms usually are found in patients older than 50 years; in a subset of patients, both neoplasms occur synchronously or metachronously. Indeed, for patients who undergo cystoprostatectomy for urothelial carcinoma of the bladder, the reported incidence of concurrent prostatic adenocarcinoma is reported to be from 46 to $70 \%(2-4)$.

At Memorial Sloan-Kettering Cancer Center (MSKCC), we frequently encounter patients with bladder neck tumors that clinically and pathologically are not clearly urothelial carcinoma or prostatic adenocarcinoma. Cystoscopy typically reveals a mass at the bladder neck, whereas the remainder of the bladder is unremarkable. Biopsies show a tumor that is often high grade and has morphologic features of both urothelial carcinoma and prostatic adenocarcinoma.

The purpose of this study was to compare the immunohistochemical (IHC) profile of intermediategrade with that of high-grade prostatic adenocarcinoma. The purpose was also to compare the IHC profile of high-grade prostatic adenocarcinoma with that of high-grade urothelial carcinoma. The latter was done in an attempt to determine an IHC panel, which enables us to distinguish prostatic adenocarcinoma from urothelial carcinoma. 


\section{MATERIALS AND METHODS}

The surgical pathology files of MSKCC were searched electronically for radical cystectomy, cystoprostatectomy, and prostatectomy specimens containing prostatic adenocarcinoma or urothelial carcinoma. Specimens subjected to neoadjuvant therapy were excluded from further study. Seventy-three examples of known prostatic adenocarcinoma and 46 examples of known urothelial carcinoma were included in this study. Prostatic adenocarcinomas were graded using the Gleason grading system $(5,6)$. All invasive urothelial carcinomas were considered to be high grade, as is the convention at our institution for locally advanced, high-stage tumors. We do not employ a grading system for invasive urothelial carcinoma, because we have found the pathologic stage and not the grade of the tumor to be an important predictor of outcome (unpublished data).

Tissue sections were assessed for high-grade areas that were then matched to the corresponding formalin-fixed, paraffin-embedded tissue block. One 3-mm punch biopsy was obtained of high-grade tumor from each tissue block, and the punch biopsies were then re-embedded as tissue multiblocks. Each multiblock contained 20 to 24 tumor samples. We chose multiblocks for this study because each punch biopsy approximates the amount of tumor seen in routine needle biopsies or transurethral resections, and the multiblocks allow for many tumor samples to be analyzed on a single slide. The multiblock technique has been shown previously to be a useful, low cost method for studying IHC antibodies $(7,8)$.

From each multiblock, $4-\mu \mathrm{m}$ sections were stained with hematoxylin and eosin (H\&E), mucicarmine, and the 11 IHC stains listed in Table 1. The immunohistochemical reactions were performed using the streptavidin-biotin horseradish peroxidase conjugation method, as described previously (9), and the antigen retrieval methods as listed in Table 1.

The IHC reactions were scored as positive for any percentage of cell reactivity. The p53 and mucicarmine stains were scored differently. For p53, at least $20 \%$ of the tumor cell nuclei had to stain to be considered positive. For mucicarmine, only intracytoplasmic staining was scored as positive. Staining intensity was scored as weak or strong with all antibodies. The intensity was considered strong if immunoreactivity could be detected at $10 \times$ magnification.

Statistical analyses were performed using Fisher's Exact Test. All comparisons were made at a significance level of $P<0.05$. The calculations were performed using Prism software (ver. 3.0; Graph Pad, San Diego, CA, USA).

\section{RESULTS}

The combined Gleason score distribution of our 73 cases of prostatic adenocarcinoma is shown in Table 2. Of the 73 cases, $27 \%$ were classified as Gleason score 6, 26\% as Gleason score 7, and $46 \%$ as Gleason score $\geq 8$.

\section{Intermediate- versus High-Grade Prostatic Adenocarcinoma}

We initially assessed the immunophenotype of prostatic adenocarcinoma, and compared intermediategrade prostatic adenocarcinoma (Gleason 6 \& 7) to high-grade prostatic adenocarcinoma (Gleason $\geq 8$ ) (Table 2). Carcinoembryonic antigen (CEAp; $P<0.0001$ ) and cytokeratin (CK) $7(P=0.0280)$ showed a statistically significant decrease in the amount of staining in high-grade tumors, whereas Leu M1 $(P=0.0150)$ showed significantly greater staining in high-grade tumors. Prostate-specific acid phosphatase (PSAP) and Leu 7 showed a smaller but insignificant decrease in the percentage of staining in high-grade tumors. Two cases of high-grade prostatic adenocarcinoma expressed $34 \beta \mathrm{E} 12$.

\section{High-Grade Prostatic Adenocarcinoma versus High-Grade Urothelial Carcinoma}

The immunohistochemical profile of high-grade prostatic adenocarcinoma was compared with that of urothelial carcinoma (Table 3). Prostate-specific

Table 1. Immunohistochemical Antibodies

\begin{tabular}{llll}
\hline \multicolumn{1}{c}{ Antibody } & Clonality & Antigen & Manufacturer \\
& retrieval & Dako (Carpinteria, CA) \\
CK 7 & Monoclonal & Pronase & Dako \\
CK 20 & Monoclonal & Protease 24 & Dako \\
$34 \beta$ E12 & Monoclonal & Protease 24 & Dako \\
Leu M1 (CD15) & Monoclonal & None & Dako \\
CEAm & Monoclonal & Citric acid & Dako \\
CEAp & Polyclonal & Trypsin & Becton Dickinson (Mountain View, CA) \\
p53 (D07) & Monoclonal & Citric acid & Becton Dickinson \\
Leu 7 (HNK-1) & Monoclonal & Pronase & Biogenex (San Ramon, CA) \\
PSAP & Monoclonal & None & Biogenex \\
PSA & Monoclonal & Citric acid & Citric acid \\
B72.3 & Monoclonal & &
\end{tabular}


Table 2. Immunophenotype of Intermediate- and High-Grade Prostate Cancer.

\begin{tabular}{|c|c|c|c|c|c|c|c|c|c|c|c|}
\hline \multirow[b]{2}{*}{ Gleason score } & \multicolumn{11}{|c|}{ No. positive (\%) } \\
\hline & PSA & PSAP & Leu 7 & CEAm & CEAp & CK 7 & CK 20 & $34 \beta \mathrm{E} 12$ & B72.3 & $\begin{array}{l}\text { Leu } \\
\text { M1 }\end{array}$ & p53 \\
\hline $6(n=20)$ & 19 (95) & $20(100)$ & $20(100)$ & $1(5)$ & $19(95)$ & $4(20)$ & $5(25)$ & 0 & $9(45)$ & $2(10)$ & 0 \\
\hline $7(n=19)$ & $19(100)$ & $19(100)$ & $19(100)$ & 0 & $19(100)$ & $10(53)$ & $6(32)$ & 0 & $11(58)$ & $1(5)$ & 0 \\
\hline$\geq 8(n=34)$ & $32(94)$ & $32(94)$ & $32(94)$ & $4(12)$ & $16(47)$ & $4(12)$ & $8(24)$ & $2(6)$ & $18(53)$ & $11(32)$ & $1(3)$ \\
\hline
\end{tabular}

PSA, prostate-specific antigen; PSAP, prostate-specific acid phosphatase; CEA, carcinoembryonic antigen; CK, cytokeratin.

Table 3. Immunophenotype of High-Grade Urothelial Carcinoma and High-Grade Prostatic Adenocarcinoma.

\begin{tabular}{|c|c|c|c|c|c|c|c|c|c|c|c|}
\hline & \multicolumn{11}{|c|}{ No. positive (\%) } \\
\hline & PSA & PSAP & Leu 7 & CEAm & CEAp & CK 7 & CK 20 & $34 \beta \mathrm{E} 12$ & B72.3 & $\begin{array}{l}\text { Leu } \\
\text { M1 }\end{array}$ & p53 \\
\hline $\mathrm{UCa}(n=46)$ & 0 & 0 & $8(17)$ & $19(41)$ & $29(63)$ & $38(83)$ & $10(22)$ & $30(65)$ & $20(43)$ & $14(30)$ & $15(33)$ \\
\hline $\mathrm{PCa}(n=34)$ & $32(94)$ & $32(94)$ & $32(94)$ & $4(12)$ & $16(47)$ & $4(12)$ & $8(24)$ & $2(6)$ & $18(53)$ & $11(32)$ & $1(3)$ \\
\hline
\end{tabular}

PSA, prostate-specific antigen; PSAP, prostate-specific acid phosphatase; CEA, carcinoembryonic antigen; CK, cytokeratin; UCa, urothelial carcinoma; $\mathrm{PCa}$, prostatic adenocarcinoma.

antigen (PSA) and PSAP only stained prostatic adenocarcinoma. Leu 7 also stained a significant percentage of prostatic adenocarcinoma, as well as a smaller percentage of urothelial carcinoma. This was found to be statistically significant $(P<0.0001)$. $34 \beta E 12$, CK 7, and p53 were found to be preferentially expressed by urothelial carcinoma $(P<$ 0.0001 ). CEAm was also preferentially expressed by urothelial carcinoma, but to a less significant degree $(P=0.0055)$. No significant difference was seen between high-grade prostatic adenocarcinoma and urothelial carcinoma in the expression of CEAp, CK 20, B72.3, Leu M1, or mucicarmine.

PSA, PSAP, Leu 7, 34 $\beta E 12$, CK 7, and p53 were determined to be the most useful antibodies for distinguishing between high-grade prostatic adenocarcinoma from high-grade urothelial carcinoma. PSA, PSAP, and Leu 7 were more often expressed in prostatic adenocarcinoma. On the other hand, 34ßE12, CK 7, and p53 were more often expressed in urothelial carcinoma. The sensitivity and specificity for each antibody are shown in Tables 4 and 5.

We also evaluated the staining intensity for these six antibodies (Tables $4 \& 5$ ). The majority of prostatic adenocarcinoma that expressed PSA, PSAP, and Leu 7 showed strong reactivity with these an-

Table 4. Sensitivity, Specificity, and Staining Intensity of Immunohistochemical Antibodies in High-Grade Prostatic Adenocarcinoma.

\begin{tabular}{lcccc}
\hline \multirow{2}{*}{ Antibody } & \multirow{2}{*}{ Sensitivity } & Specificity & \multicolumn{2}{c}{ Staining Intensity } \\
\cline { 5 - 6 } & & Strong & Weak \\
\hline & & $\%$ & & \\
PSA & 95.8 & 100 & 68.8 & 31.2 \\
PSAP & 97.2 & 100 & 84.4 & 15.6 \\
Leu 7 & 97.2 & 82.6 & 96.9 & 3.1 \\
\hline
\end{tabular}

PSA, prostate-specific antigen; PSAP, prostate-specific acid phosphatase.
Table 5. Sensitivity, Specificity, and Staining Intensity of Immunohistochemical Antibodies in High-Grade Urothelial Carcinoma

\begin{tabular}{lcccc}
\hline \multirow{2}{*}{ Antibody } & \multirow{2}{*}{ Sensitivity } & Specificity & \multicolumn{2}{c}{ Staining Intensity } \\
\cline { 5 - 5 } & & & Strong & Weak \\
\hline $34 \beta$ E12 & 65.2 & 97.2 & 83.3 & 16.6 \\
CK 7 & 82.6 & 75.3 & 36.8 & 55.2 \\
p53 & 32.6 & 98.6 & 80 & 20 \\
\hline
\end{tabular}

CK, cytokeratin.

tibodies. Most urothelial carcinoma that reacted with $34 \beta \mathrm{E} 12$ demonstrated strong positivity, whereas expression of CK 7 was weak for the majority of the tumors.

Several of the known cases of prostatic adenocarcinoma and urothelial carcinoma showed unexpected results. One case of prostatic adenocarcinoma was negative for both PSA and PSAP; it was also negative for CK 7 and $34 \beta E 12$ but positive for Leu 7 (Figure 1). Two other cases of prostatic adenocarcinoma were negative for Leu 7 but positive for PSA and/or PSAP. With regard to urothelial carcinoma, 4 cases showed no reactivity for CK 7 , $34 \beta E 12$, PSA, PSAP, or Leu 7 . Two of these four cases were positive for p53 (Figure 2, A and B). Eight urothelial carcinomas expressed Leu 7, but also expressed CK 7 and/or $34 \beta E 12$. All seven of these cases were negative for PSA and PSAP.

\section{DISCUSSION}

Our results show that a panel of six IHC antibodies is useful in distinguishing high-grade prostatic adenocarcinoma from urothelial carcinoma. These antibodies include PSA, PSAP, Leu $7,34 \beta E 12$, CK 7 , and p53. A positive result with the antibodies listed in Table 4 supports a diagnosis of prostatic adeno- 


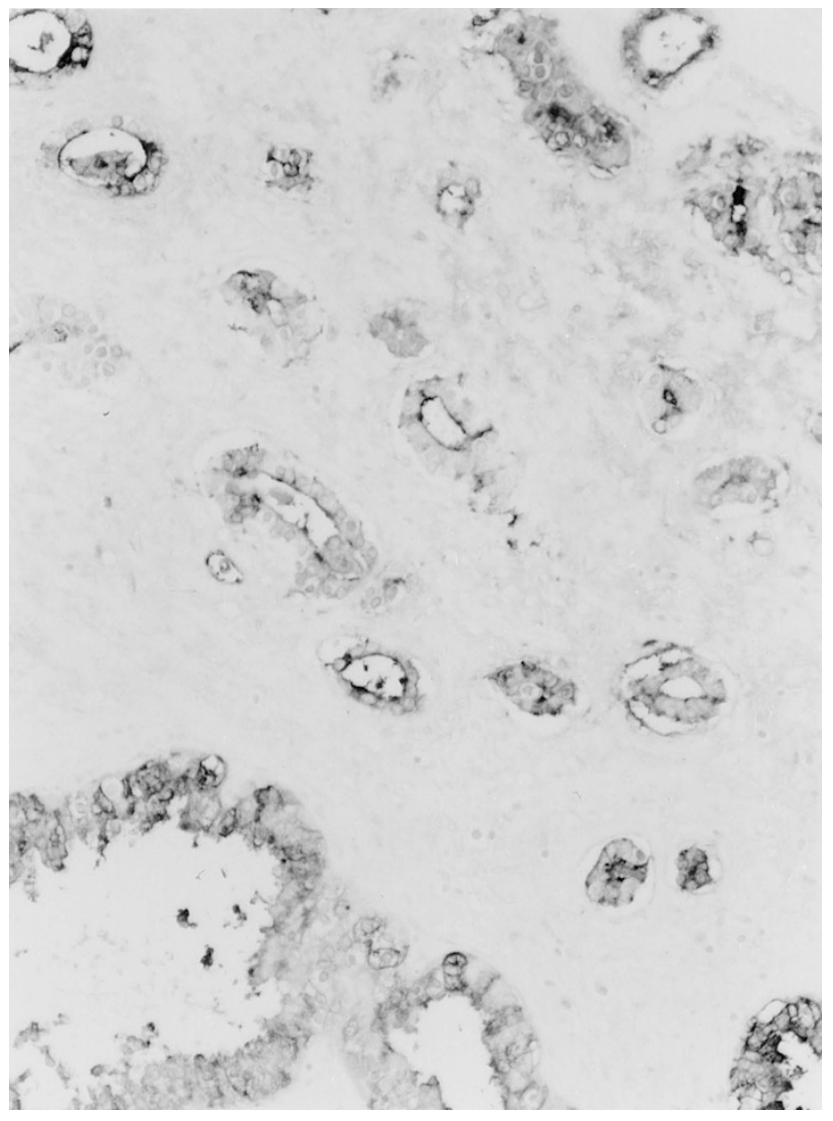

FIGURE 1. Prostatic adenocarcinoma: Positive staining of benign duct and tumor cells with Leu 7.

carcinoma, whereas a positive result with an antibody in Table 5 supports a diagnosis of urothelial carcinoma.

Our study supports the results of others showing PSA and PSAP to be highly sensitive and specific for prostatic adenocarcinoma (10-12). Indeed, we found both PSA and PSAP to be $100 \%$ specific for prostatic adenocarcinoma. Like others, we found a rare case of high-grade prostatic adenocarcinoma that was negative for both PSA and PSAP $(10,11$, 13-15). Similar to previous reports, none of our cases of urothelial carcinoma of the urinary bladder expressed PSA or PSAP $(11,12)$.

We found Leu 7 to be a sensitive and relatively specific marker of prostatic adenocarcinoma when compared with urothelial carcinoma. Leu 7 was initially characterized as a human natural killer cellbinding antibody and subsequently was found to bind a variety of carcinomas including prostatic adenocarcinoma (16-19). May et al. (17) reported consistent Leu 7 immunoreactivity in primary and metastatic prostatic adenocarcinoma, and Wahab and Wright (19) found Leu 7 immunoreactivity in $95 \%$ of prostatic adenocarcinomas. In contrast, we found only $17 \%$ of urothelial carcinomas to be Leu 7-positive.

In contrast to previous studies, we found only a minority urothelial carcinomas to be CK 20 positive
(20-22). Reasons for this apparent discrepancy between studies is unclear. Wang et al. (22) reported CK 20 expression in 17 of 19 urothelial carcinomas (89\%) and used a cutoff similar to ours (more than $1 \%$ positive cells). Also using a percentage cutoff similar to ours, Miettinen (20) found CK 20 expression in $58 \%$ of urothelial carcinomas. Like Wang et al. (22), we found the majority of urothelial carcinomas and a minority of prostatic adenocarcinomas to be positive for CK 7 .

Our data show that the high-molecular-weight cytokeratin antibody $34 \beta \mathrm{E} 12$ is an insensitive but highly specific marker in distinguishing urothelial carcinoma from prostatic adenocarcinoma. $34 \beta \mathrm{E} 12$ binds to high-molecular-weight cytokeratins 1,5 , 10 , and 14 and has been previously shown to be highly expressed in urothelial carcinoma and only rarely expressed in prostatic adenocarcinoma (23, 24). It is most commonly used to label prostate basal cells and thus distinguish benign prostate lesions from prostatic adenocarcinoma (25-27). Recently, Googe et al. (28) reported a high frequency of $34 \beta \mathrm{E} 12$ expression in primary and metastatic prostatic adenocarcinoma. However, our data and that of Yang et al. (24), which collectively includes 183 cases of prostate cancer, shows that $34 \beta E 12$ expression is rare in prostatic adenocarcinoma.

Similar to previous studies, we found almost half of the cases of high grade prostate and urothelial carcinomas ( $53 \%$ and $43 \%$, respectively) to be immunoreactive to B72.3, a monoclonal antibody that binds tumor-associated glycoprotein-72 (TAG-72) (29-33). Although Mazur et al. (31) found that the majority of prostatic adenocarcinomas were B72.3 positive, most were either weakly or focally positive. Myers et al. (32), on the other hand, found that $31 \%$ of prostatic adenocarcinomas were "moderately" or "strongly" B72.3 positive.

The monoclonal antibody Leu M1 is a myelomonocytic cell marker that has been reported to stain a subset of urothelial carcinomas, particularly in situ carcinoma (34). For example, Hoshi et al. (34) found Leu M1 expression in 50\% of in situ urothelial carcinomas compared to only $7 \%$ invasive carcinomas. We, however, found $30 \%$ of invasive urothelial carcinomas to be Leu M1 positive.

Similar to previous studies, we found CEAp to be commonly expressed in urothelial carcinoma (3537). However, because CEAp is also frequently expressed in prostatic adenocarcinoma, it is not useful in distinguishing prostatic adenocarcinoma from urothelial carcinoma (13). We found urothelial carcinomas to express CEAm more often than prostate carcinomas.

In a subset of cases, p53 immunohistochemistry may help to distinguish urothelial carcinoma from prostatic adenocarcinoma. Mutations of the p53 gene, as determined by p53 immunohistochemis- 

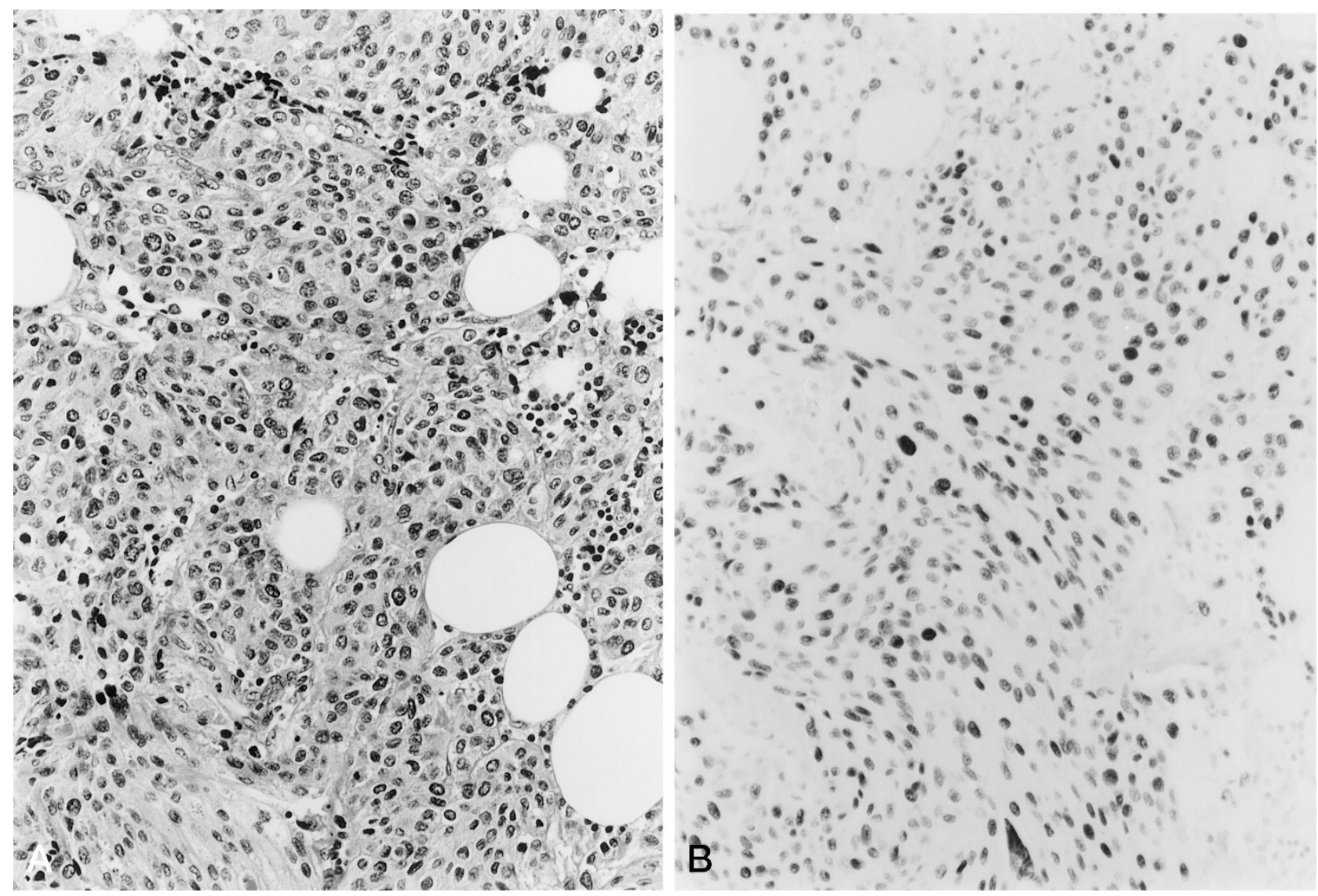

FIGURE 2. Urothelial carcinoma: A, high grade urothelial carcinoma involving perivesical adipose tissue. B, tumor nuclei show immunoreactivity with p53.

try, are common in invasive urothelial carcinoma and androgen-independent prostatic adenocarcinoma but are uncommon in hormone-sensitive, localized prostatic adenocarcinoma. For example, p53 accumulation or overexpression has been reported in 33 to $81 \%$ of urothelial carcinomas, $60 \%$ of androgen-independent prostatic adenocarcinomas, and 1 to $19 \%$ of hormone-sensitive prostatic adenocarcinomas (38-41).

In conclusion, we describe an immunohistochemical panel including PSA, PSAP, Leu $7,34 \beta E 12$, CK 7, and p53, that may be helpful in distinguishing high-grade prostatic adenocarcinoma from high grade urothelial carcinoma in diagnostically difficult cases. A positive stain with PSA or PSAP confirms the diagnosis of prostate carcinoma, and a positive stain with Leu 7 favors this same diagnosis. Currently, there are no markers entirely specific for urothelial carcinoma; however, a positive reaction with $34 \beta \mathrm{E} 12$, CK 7 , or p53 supports the diagnosis of urothelial carcinoma. We recommend initially performing PSA, PSAP, and $34 \beta E 12$. In the event that these three stains are negative, Leu 7, CK 7, and p53 could be used.

Acknowledgment: The authors thank Mr. Kin Chung Kong and Ms. Allyne D. Manzo of the Memo- rial Sloan-Kettering Cancer Center for their assistance in the preparation of the photomicrographs.

\section{REFERENCES}

1. Landis SH, Murray T, Bolden S, Wingo PA. Cancer statistics, 1998 [published erratum appears in CA Cancer J Clin 1998; 48:192]. CA Cancer J Clin 1998;48:6-29.

2. Mahadevia PS, Koss LG, Tar IJ. Prostatic involvement in bladder cancer. Prostate mapping in 20 cystoprostatectomy specimens. Cancer 1986;58:2096-102.

3. Montie JE, Wood DPJ, Pontes JE, Boyett JM, Levin HS. Adenocarcinoma of the prostate in cystoprostatectomy specimens removed for bladder cancer. Cancer 1989;63:381-5.

4. Troncoso P, Babaian RJ, Ro JY, Grignon DJ, von Eschenbach AC, Ayala AG. Prostatic intraepithelial neoplasia and invasive prostatic adenocarcinoma in cystoprostatectomy specimens. Urology 1989;34:52-6.

5. Gleason DF. Histologic grading of prostate cancer: a perspective. Hum Pathol 1992;23:273-9.

6. Gleason DF, Mellinger GT. Prediction of prognosis for prostatic adenocarcinoma by combined histological grading and clinical staging. J Urol 1974;111:58-64.

7. Battifora H. The multitumor (sausage) tissue block: novel method for immunohistochemical antibody testing. Lab Invest 1986;55:244-8.

8. Kraaz W, Risberg B, Hussein A. Multiblock: an aid in diagnostic immunohistochemistry [letter]. J Clin Pathol 1988;41: 1337.

9. Cartun RW, Pedersen CA. An immunocytochemical technique offering increased sensitivity and lowered cost with a 
streptavidin-horseradish peroxidase conjugate. J Histotechnol 1989;12:273-7.

10. Bentz MS, Cohen C, Demers LM, Budgeon LR. Immunohistochemical demonstration of prostatic origin of metastases. Urology 1982;19:584-6.

11. Jobsis AC, De Vries GP, Anholt RR, Sanders GT. Demonstration of the prostatic origin of metastases: an immunohistochemical method for formalin-fixed embedded tissue. Cancer 1978;41:1788-93.

12. Nadji M, Tabei SZ, Castro A, Chu TM, Murphy GP, Wang MC, Morales AR. Prostatic-specific antigen: an immunohistologic marker for prostatic neoplasms. Cancer 1981;48:1229-32.

13. Ellis DW, Leffers S, Davies JS, Ng AB. Multiple immunoperoxidase markers in benign hyperplasia and adenocarcinoma of the prostate. Am J Clin Pathol 1984;81:279-84.

14. Stein BS, Vangore S, Petersen RO, Kendall AR. Immunoperoxidase localization of prostate-specific antigen. Am J Surg Pathol 1982;6:553-7.

15. Epstein JI. PSA and PAP as immunohistochemical markers in prostate cancer. Urol Clin North Am 1993;20:757-70.

16. Abo T, Balch CM. A differentiation antigen of human NK and K cells identified by a monoclonal antibody (HNK-1). J Immunol 1981;127:1024-9.

17. May EE, Perentes E Anti-Leu 7 immunoreactivity with human tumours: its value in the diagnosis of prostatic adenocarcinoma. Histopathology 1987;11:295-304.

18. Rusthoven JJ, Robinson JB, Kolin A, Pinkerton PH. The natural-killer-cell-associated HNK-1 (Leu-7) antibody reacts with hypertrophic and malignant prostatic epithelium. Cancer 1985;56:289-93.

19. Wahab ZA, Wright GLJ. Monoclonal antibody (anti-Leu 7) directed against natural killer cells reacts with normal, benign and malignant prostate tissues. Int J Cancer 1985;36: 677-83.

20. Miettinen M. Keratin 20: immunohistochemical marker for gastrointestinal, urothelial, and Merkel cell carcinomas. Mod Pathol 1995;8:384-8.

21. Moll R, Lowe A, Laufer J, Franke WW. Cytokeratin 20 in human carcinomas. A new histodiagnostic marker detected by monoclonal antibodies. Am J Pathol 1992;140:427-47.

22. Wang NP, Zee S, Zarbo RJ, Bacchi CE, Gown AM. Coordinate expression of cytokeratins 7 and 20 defines unique subsets of carcinomas. Appl Immunohistochem 1995;3:99-107.

23. Gown AM, Vogel AM. Monoclonal antibodies to human intermediate filament proteins. III. Analysis of tumors. Am J Clin Pathol 1985;84:413-24.

24. Yang XJ, Lecksell K, Gaudin P, Epstein JI. Rare expression of high-molecular-weight cytokeratin in adenocarcinoma of the prostate gland: a study of 100 cases of metastatic and locally advanced prostate cancer. Am J Surg Pathol 1999;23:147-52.

25. Hedrick L, Epstein JI. Use of keratin 903 as an adjunct in the diagnosis of prostate carcinoma. Am J Surg Pathol 1989;13: 389-96.

26. Brawer MK, Peehl DM, Stamey TA, Bostwick DG. Keratin immunoreactivity in the benign and neoplastic human prostate. Cancer Research 1985;45:3663-67.

27. Wojno KJ, Epstein JI. The utility of basal cell-specific anti- cytokeratin antibody (34 beta E12) in the diagnosis of prostate cancer. A review of 228 cases. Am J Surg Pathol 1995;19: 251-60.

28. Googe PB, McGinley KM, Fitzgibbon JF. Anticytokeratin antibody 34 beta E12 staining in prostate carcinoma. Am J Clin Pathol 1997;107:219-23.

29. Brenner PC, Rettig WJ, Sanz-Moncasi MP, Reuter V, Aprikian A, Old LJ, Fair WR, Garin-Chesa P. TAG-72 expression in primary, metastatic and hormonally treated prostate cancer as defined by monoclonal antibody CC49 [see comments]. J Urol 1995;153:1575-9.

30. Loy TS, Nashelsky MB. Reactivity of B72.3 with adenocarcinomas. An immunohistochemical study of 476 cases. Cancer 1993;72:2495-8.

31. Mazur MT, Shultz JJ. Prostatic adenocarcinoma. Evaluation of immunoreactivity to monoclonal antibody B72.3. Am J Clin Pathol 1990;93:466-70.

32. Myers RB, Meredith RF, Schlom J, Lo BA, Bueschen AJ, Wheeler RH, Stockard CR, Grizzle WE. Tumor associated glycoprotein-72 is highly expressed in prostatic adenocarcinomas. J Urol 1994;152:243-6.

33. Myers RB, Schlom J, Srivastava S, Grizzle WE. Expression of tumor-associated glycoprotein 72 in prostatic intraepithelial neoplasia and prostatic adenocarcinoma. Mod Pathol 1995; 8:260-5.

34. Hoshi S, Orikasa S, Numata I, Nose M. Expression of Leu-M1 antigens in carcinoma of the urinary bladder. J Urol 1986; 135:1075-7.

35. Heyderman E, Brown BM, Richardson TC. Epithelial markers in prostatic, bladder, and colorectal cancer: an immunoperoxidase study of epithelial membrane antigen, carcinoembryonic antigen, and prostatic acid phosphatase. J Clin Pathol 1984;37:1363-9.

36. Jautzke G, Altenaehr E. Immunohistochemical demonstration of carcinoembryonic antigen (CEA) and its correlation with grading and staging on tissue sections of urinary bladder carcinomas. Cancer 1982;50:2052-6.

37. Shevchuk MM, Fenoglio CM, Richart RM. Carcinoembryonic antigen localization in benign and malignant transitional epithelium. Cancer 1981;47:899-905.

38. Aprikian AG, Sarkis AS, Fair WR, Zhang ZF, Fuks Z, CordonCardo C. Immunohistochemical determination of p53 protein nuclear accumulation in prostatic adenocarcinoma. J Urol 1994;151:1276-80.

39. Dalbagni G, Presti JCJ, Reuter VE, Zhang ZF, Sarkis AS, Fair WR, Cordon-Cardo C. Molecular genetic alterations of chromosome 17 and p53 nuclear overexpression in human bladder cancer. Diagn Mol Pathol 1993;2:4-13.

40. Sarkis AS, Dalbagni G, Cordon-Cardo C, Zhang ZF, Sheinfeld J, Fair WR, Herr HW, Reuter VE. Nuclear overexpression of p53 protein in transitional cell bladder carcinoma: a marker for disease progression. J Natl Cancer Inst 1993;85:53-9.

41. Sidransky D, Von Eschenbach A, Tsai YC, Jones P, Summerhayes I, Marshall F, Paul M, Green P, Hamilton SR, Frost P. Identification of p53 gene mutations in bladder cancers and urine samples. Science 1991;252:706-9. 